\title{
A continuum approach to electron solvation by a layer of polar adsorbates
}

\author{
K. L. Sebastian ${ }^{\text {a) }}$ \\ National Institute of Advanced Industrial Science and Technology, AIST Central 5, 1-1 Higashi, \\ Tsukaba, Ibaraki 305-8565, Japan and Department of Inorganic and Physical Chemistry, \\ Indian Institute of Science, Bangalore 560012, India \\ Aniruddha Chakraborty \\ Department of Inorganic and Physical Chemistry, Indian Institute of Science, Bangalore 560012, India \\ M. Tachiya ${ }^{\text {b) }}$ \\ National Institute of Advanced Industrial Science and Technology, AIST Central 5, 1-1 Higashi, Tsukaba, \\ Ibaraki 305-8565, Japan
}

\begin{abstract}
We give a continuum description of the solvation of an electron in the image state of a metal surface by a layer of polar adsorbates. In the model, we account for the interaction of the dipole moment of the adsorbate with the electric field exerted by the electron, which is perpendicular to the surface. We also include the dipolar interactions between the adsorbates. With this simple model, it is easy to make an analysis of the self-trapping of electron. Depending upon the values of the parameters, the self-trapped state can have any arbitrary size. Also, there are regimes in which (1) there is no localized state, (2) a localized and delocalized state coexist, with the delocalized state being a saddle point on the potential energy surface, and the localized state a minimum and (3) both the states exist as stable minima, and there is a barrier between the two. In the second case, self-trapping would be a barrierless process while for the third, it would be an activated process. We find that our model can explain the salient features of the experimental results of Harris et al. [Science 297, 1163 (2002)]. At the parameter value required to fit the experimental data, self-trapping is barrierless.
\end{abstract}

\section{INTRODUCTION}

An electron near a metal surface feels the charge of its image in the metal. Consequently near a metal surface it moves under the influence of this Coulombic attractive potential, and there can be quantized energy levels which lie in the band gap of the metal and whose wave functions lie outside the surface. ${ }^{1-3}$ These image potential states can be studied using two photon photoemission spectroscopy, ${ }^{2}$ which is a sensitive and valuable tool for the study of relaxation processes at interfaces. Harris et al. have carried out interesting studies of image potential states $^{4-8}$ using this technique.

Recently, in a very interesting paper Harris et al. ${ }^{4}$ report an experimental study of the ultrafast electron solvation at two-dimensional metal/polar adsorbate interface. They used two-photon photoemission to study the electrons in image states of $\operatorname{Ag}(111)$ surface having polar adsorbates on it. The study is interesting because there are interactions between the electron and polarization modes of the adsorbate. While such polarization interactions are well studied in the bulk, very little is known about them at an interface. In the bulk there is the possibility of trapping of the electron in the selfinduced potential. In their study of the interface, Harris et al.

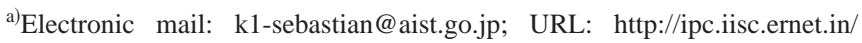
$\sim \mathrm{kls} /$

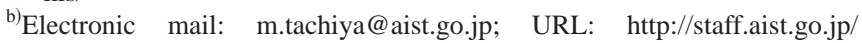
m.tachiya/
}

find two kinds of states, viz., one delocalized and the other, localized. On the theoretical side, the localized (self-trapped) state of an electron/exciton in a $D$-dimensional lattice has been a subject of a large body of literature (for example, see Refs. 9-17, and the references therein). In two dimensions, with a continuum model for the lattice with short range interaction with the electron, it was found that if the coupling constant exceeds a critical value, then the electron localizes itself to a state of spatial extension roughly of the size of lattice spacing. ${ }^{15,16}$ In the experiment, ${ }^{4}$ it is found that the localized state has roughly the size of an adsorbed molecule. This system is a very interesting one in that the electronic wave function is spread out in three dimensions and is in interaction with a two-dimensional layer of polar molecules. As there does not seem to be any theoretical study of this problem, we investigate the following: (1) What are the conditions for the existence of a localized state? (2) What are the dimensions of the localized state? Is it necessary that it should be localized to a region of the size of a molecule, or is it possible to have states which sit over a larger area? (3) For a given set of parameters, can one have both localized and delocalized states as extrema? (4) If both exist as possible extrema, is there a barrier separating the two? Or, in other words, is the delocalized state stable with respect to deformations? The use of a continuum model allows us to carry out the analysis rather easily. In the bulk, continuum models have been used with success long ago. ${ }^{18,19}$ 


\section{THE MODEL}

The simplest possible Hamiltonian that one can use for the description of the system is described below. It is a continuum one and would be valid for the early stages of the solvation, when the electron is still delocalized over larger distances, and may break down in the late stages, if the electron localizes itself into a state which is only of molecular dimensions. In spite of this, a continuum model would be useful for qualitative understanding of the process and should serve as a starting point for more detailed studies, which takes discreteness of the adsorbate into account. Further, in contrast to electrons in bulk liquids, the electron at the interface extends out into the vacuum. Therefore, its interaction with the polar adsorbate is much weaker, and unlike in the bulk, the electron is not trapped in between a few of the polar molecules and therefore, our methodology is reasonable. Our approach has the following ingredients:

(1) Polarization of the adsorbed layer, perpendicular to the surface, which we denote by $P(s, t)$. Here $s$ is a twodimensional vector, specifying position within the layer on the surface. The polarization parallel to the surface do not play any role in the solvation, because the electric field due to the electron at the surface of the metal is perpendicular to it. This polarization has an initial value zero, at the time when the electron is put into the image state. But then, because of the electric field of the electron, the polarization slowly builds up and the electron gets more and more localized and its energy is lowered and it gets trapped. In the continuum limit, the polarization may be described by the following expression for its energy:

$E_{\mathrm{pol}}=\frac{\rho}{2} \int d \boldsymbol{s} \dot{P}(s, t)^{2}+\frac{\rho \omega_{0}^{2}}{2} \int d \boldsymbol{s} P(\boldsymbol{s}, t)^{2}+E_{\mathrm{dipolar}}$,

$\dot{P}(s, t)=d P(s, t) / d t . \rho$ is a constant (see Appendix A for justification of this expression). Each molecule in the layer can reorient and thus the layer can get polarized by the charge of the electron. The angular frequency of the reorientational motion is taken to be $\omega_{0}$. In addition, there is dipolar interaction between polarizations at different locations and we shall call this energy $E_{\text {dipolar }}$. In the continuum limit, one has to be careful in writing down this term. Remembering that $P(s, t)$ is in the direction perpendicular to the surface, we write this as

$E_{\text {dipolar }}=\int_{\left|s-s^{\prime}\right|>a} d s \int d \boldsymbol{s}^{\prime} P(s, t) \frac{1}{\left|\boldsymbol{s}-\boldsymbol{s}^{\prime}\right|^{3}} P\left(\boldsymbol{s}^{\prime}, t\right)$.

In the above, $a$ is a lower cut-off, roughly of the order of lattice spacing, which prevents self interaction. The above expression also includes the interaction of the dipoles with their own images. This expression accounts for dipole-dipole interaction in the simplest possible way (see Appendix A for details). The condition $\left|s-s^{\prime}\right|$ $>a$ in the above integral can be easily taken into account by modifying the integral to

$E_{\text {dipolar }}=\int d \boldsymbol{s} \int d \boldsymbol{s}^{\prime} P(\boldsymbol{s}, t) \frac{1}{\left(\left|\boldsymbol{s}-\boldsymbol{s}^{\prime}\right|^{2}+a^{2}\right)^{3 / 2}} P\left(\boldsymbol{s}^{\prime}, t\right)$.

The error caused by this modification can be corrected for by subtracting a term proportional to $\int d s P(s, t)^{2}$ from the total energy and this will only lead to a redefining of the value of $\omega_{0}^{2}$, which we assume has been done, and we shall denote the redefined value by the same symbol. Using the above, the polarization energy, $E_{\mathrm{pol}}$ can be written in terms of the Fourier components defined by $P(\boldsymbol{k}, t)=(1 / 2 \pi) \int d \boldsymbol{s} P(\boldsymbol{s}, t) e^{i \boldsymbol{k} . \boldsymbol{s}}$ as the quadratic functional,

$$
\begin{aligned}
E_{\mathrm{pol}}= & \frac{\rho}{2} \int d \boldsymbol{k}|\dot{P}(\boldsymbol{k}, t)|^{2}+\frac{\rho \omega_{0}^{2}}{2} \int d \boldsymbol{k}|P(\boldsymbol{k}, t)|^{2} \\
& +\frac{2 \pi}{a} \int d \boldsymbol{k}|P(\boldsymbol{k}, t)|^{2} e^{-k a} .
\end{aligned}
$$

Note that the wave vector $\boldsymbol{k}$ is two-dimensional and $k$ $=|\boldsymbol{k}|$. This expression for energy can be taken to be the limit of the expression for energy of a discrete model, as discussed in Appendix A. Strictly speaking, one should also include the parallel component of the polarization, too in the description, because the adlayer is two molecules thick and the molecules that do reorient, in presence of the electron are in the second layer and hence are not actually on the surface of the metal. Away from the surface, the electric field is expected to have a parallel component too, but one expects its effect to be small in comparison with that of the perpendicular electric field. We shall neglect this parallel field, as it simplifies our analysis considerably.

(2) The electronic part of the Hamiltonian is given by (we use atomic units, and put $\hbar=1, e=1$ and $m_{e}=1$ )

$H_{\mathrm{el}}=-\frac{1}{2} \nabla^{2}-\frac{1}{4 z}+V_{\mathrm{hw}}$.

- $(1 / 4 z)$ comes from the image interaction, while $V_{\mathrm{hw}}$ is from the interaction of the electron with the surface, which we assume is given by a hard wall like term. We take the hard wall to be located at a height $h$ from the surface of the metal.

(3) Finally, one has the interaction between the electron and the polarization of the layer. The interaction energy is

$E_{\mathrm{int}}=-\int d \boldsymbol{s} E(\boldsymbol{r}, \boldsymbol{s}) P(\boldsymbol{s}, t)$.

$E(r, s)$ is the electric field at the point $\boldsymbol{s}$ on the surface, due to an electron at $\boldsymbol{r}$ and its image in the metal. In terms of the Fourier components, the interaction energy may be written as

$$
E_{\text {int }}=-2 \int d \boldsymbol{k} \exp \left(i \boldsymbol{k} \cdot \boldsymbol{r}_{\|}\right) P^{*}(\boldsymbol{k}, t) \exp (-k z) .
$$

$\boldsymbol{r}_{\|}(\equiv(x, y))$ is the component of $\boldsymbol{r}$ parallel to the surface.

\section{THE POTENTIAL ENERGY SURFACE}

Our strategy is as follows: We use a trial wave function for the electron, containing two unknown parameters $\alpha$ and $\beta$, which determine the extent of the wave function in the parallel and perpendicular directions. Using this trial function we calculate the expectation value of the energy. We then find polarization such that the resultant expression for energy has the least value. Using this optimum polarization, 
given in Eq. (13) we calculate the minimum energy as a function of the parameters $\alpha$ and $\beta$. Finally, we find those values of $\alpha$ and $\beta$ which makes this energy a minimum.

Our trial function is

$$
\psi=\left(\frac{\alpha^{2} \beta^{3}}{2 \pi}\right)^{1 / 2} \exp \left(-\alpha^{2} \boldsymbol{r}_{\|}^{2} / 2-\beta(z-h) / 2\right)(z-h) .
$$

The term $(z-h)$ ensures that the hard wall condition is satisfied at $z=h$. Using this, we calculate the expectation value of the electronic part and the interaction energy in Eq. (9). This gives the total energy as

$$
\begin{aligned}
E= & \frac{1}{2} \alpha^{2}+\epsilon_{\mathrm{el}}(\beta)+\frac{\rho}{2} \int d \boldsymbol{k}|\dot{P}(\boldsymbol{k})|^{2}+\frac{1}{2} \int d \boldsymbol{k}\left(\rho \omega_{0}^{2}\right. \\
& \left.+\frac{4 \pi}{a} e^{-k a}\right)|P(\boldsymbol{k})|^{2}-2 \int d \boldsymbol{k} \exp \left(-k^{2} /\left(4 \alpha^{2}\right)-h k\right) \\
& \times\left(\frac{\beta}{k+\beta}\right)^{3} P(\boldsymbol{k})^{*} .
\end{aligned}
$$

$\frac{1}{2} \alpha^{2}+\epsilon_{\mathrm{el}}(\beta)$ is the purely electronic energy, and

$$
\epsilon_{\mathrm{el}}(\beta)=\frac{1}{8}\left(-\beta+\beta^{2}+h \beta^{2}-e^{h \beta} h^{2} \beta^{3} \Gamma(0, h \beta)\right) .
$$

$\Gamma(a, z)$ is the incomplete Gamma function, defined to be equal to $\int_{z}^{\infty} t^{a-1} \exp (-t) d t$. We now define

$$
\begin{aligned}
\mathcal{E}= & \frac{1}{2} \alpha^{2}+\epsilon_{\mathrm{el}}(\beta)+\frac{1}{2} \int d \boldsymbol{k}\left(\rho \omega_{0}^{2}+\frac{4 \pi}{a} e^{-k a}\right)|P(\boldsymbol{k})|^{2} \\
& -2 \int d \boldsymbol{k} \exp \left(-k^{2} /\left(4 \alpha^{2}\right)-h k\right)\left(\frac{\beta}{k+\beta}\right)^{3} P(\boldsymbol{k})^{*} .
\end{aligned}
$$

$\mathcal{E}$ includes all the energies except, kinetic energy of the polarization. Now we look for the polarization that optimizes the energy $\mathcal{E}$ in Eq. (11). As the electronic density is assumed to have cylindrical symmetry, the induced polarization on the surface would have circular symmetry. This means that $P(\boldsymbol{k}, t)$, would depend only on the magnitude of the wave vector $\boldsymbol{k}$. With this condition, the polarization that minimizes $\mathcal{E}$ is

$$
P_{\text {opt }}(k)=2\left(\frac{\beta}{k+\beta}\right)^{3} \frac{\exp \left(-k^{2} /\left(4 \alpha^{2}\right)-h k\right)}{\left(\rho \omega_{0}^{2}+\frac{4 \pi}{a} e^{-k a}\right)} .
$$

Note that imaginary part of $P_{\text {opt }}(k)$ is zero. Using this in Eq. (11) we get the minimum energy as a function of the parameters $\alpha$ and $\beta$, and we write it as

$$
E(\alpha, \beta)=\frac{1}{2} \alpha^{2}+\epsilon_{\mathrm{el}}(\beta)-V(\alpha, \beta)
$$

with

$$
V(\alpha, \beta)=4 \pi \int_{0}^{\infty} d k k\left(\frac{\beta}{k+\beta}\right)^{6} \frac{e^{-2 h k-k^{2} / 2 \alpha^{2}}}{\left(4 \pi e^{-a k} / a+\rho \omega_{0}^{2}\right)} .
$$

$E(\alpha, \beta)$ is the expression for the minimum energy of the system, having a fixed value for the parameters $\alpha$ and $\beta$ in the wave function. Using the expression for $E(\alpha, \beta)$, we can analyze the existence of extrema, corresponding to localized/ delocalized states. If $E(\alpha, \beta)$ has an extremum, which is a minimum, then so does $\mathcal{E}$. On the other hand, if $E(\alpha, \beta)$ is unstable in one direction, then that means, on the surface for $\mathcal{E}$, there are fluctuations that will lower the energy and hence the extremum on multidimensional surface will also be unstable. From the analysis of Appendix B 1 using $E(\alpha, \beta)$, we conclude the following ( $a_{0}$ is the radius of the first Bohr orbit): (1) If $\rho a_{0} \omega_{0}^{2}$ is large, there is no localized state and delocalized state is a minimum in the PES. Therefore, selftrapping is not possible. (2) For $\rho a_{0} \omega_{0}^{2}<4 \pi\left(2-a_{0} / a\right)$, a localized state would exist and is the stable minimum. The delocalized state would also exist, and it is not a stable minimum, but a saddle. Hence self trapping is a barrierless process. (3) For $4 \pi\left(2-a_{0} / a\right)<\rho a_{0} \omega_{0}^{2}<8 \pi$ the delocalized state is a stable minimum and a localized state may or may not exist. If it exists, then it too is a minimum and there would be a barrier between the two, as result of which the self trapping is an activated process.

The energy $E(\alpha, \beta)$, while useful, is not what is usually referred to as the potential energy surface, because in the evaluation of $E(\alpha, \beta)$, we have assumed the wave function for the electron to be fixed, and adjusted the polarization so as to have the minimum energy. What is of greater interest is the surface where the electronic energy is minimized for a given polarization. We would now like to construct such a surface for the system near the trapped state. The actual surface is infinite dimensional, but we construct a surface, depending on two parameters, which are important for the description of the trapping. Thus we consider polarizations of the form,

$$
P_{w}\left(\alpha_{w}, \Delta_{w}, \boldsymbol{s}\right)=\Delta_{w} \alpha_{w}^{2} \exp \left(-\alpha_{w}^{2} \boldsymbol{s}^{2}\right)
$$

$\alpha_{w}$ determines its width of the polarization and as $\int d s P_{w}\left(\alpha_{w}, \Delta_{w}, s\right)=\pi \Delta_{w}, \Delta_{w}$ parameter determines the total integrated strength of the polarization. When one Fourier transforms, one gets $P_{w}\left(\alpha_{w}, \Delta_{w}, k\right)=\left(\Delta_{w} / 2\right) \exp \left(-k^{2} /\right.$ $\left.\left(4 \alpha_{w}^{2}\right)\right)$. For these, taking the wave function to be given by Eq. (8), we can calculate the energy to be

$$
\begin{aligned}
E\left(\alpha, \beta, \alpha_{w}, \Delta_{w}\right) & \frac{1}{2} \alpha^{2}+\epsilon_{\mathrm{el}}(\beta)+\frac{\pi \Delta_{w}^{2}}{4} \int_{0}^{\infty} k d k\left(4 \pi e^{-a k} / a+\rho \omega_{0}^{2}\right) \\
& \times \exp \left(-k^{2} /\left(2 \alpha_{w}^{2}\right)\right)-2 \pi \Delta_{w} \int_{0}^{\infty} k d k \\
& \times \exp \left\{-\frac{k^{2}}{4}\left(\frac{1}{\alpha^{2}}+\frac{1}{\alpha_{w}^{2}}\right)-h k\right\}\left(\frac{\beta}{k+\beta}\right)^{3} \\
= & \frac{1}{2} \alpha^{2}+\epsilon_{\mathrm{el}}(\beta)+\frac{\pi \alpha_{w}^{2} \Delta_{w}^{2}}{4}\left(\frac{4 \pi}{a}+\rho \omega_{0}^{2}\right) \\
& -\frac{\alpha_{w}^{3} \Delta_{w}^{2}}{\sqrt{2}} e^{a^{2} \alpha_{w}^{2} / 2} \pi^{5 / 2} \operatorname{Erfc}\left(\frac{a \alpha_{w}}{\sqrt{2}}\right)-2 \pi \Delta_{w} \int_{0}^{\infty} k d k \\
& \times \exp \left\{-\frac{k^{2}}{4}\left(\frac{1}{\alpha^{2}}+\frac{1}{\alpha_{w}^{2}}\right)-h k\right\}\left(\frac{\beta}{k+\beta}\right)^{3} \cdot
\end{aligned}
$$

Now we can define a potential energy surface by 


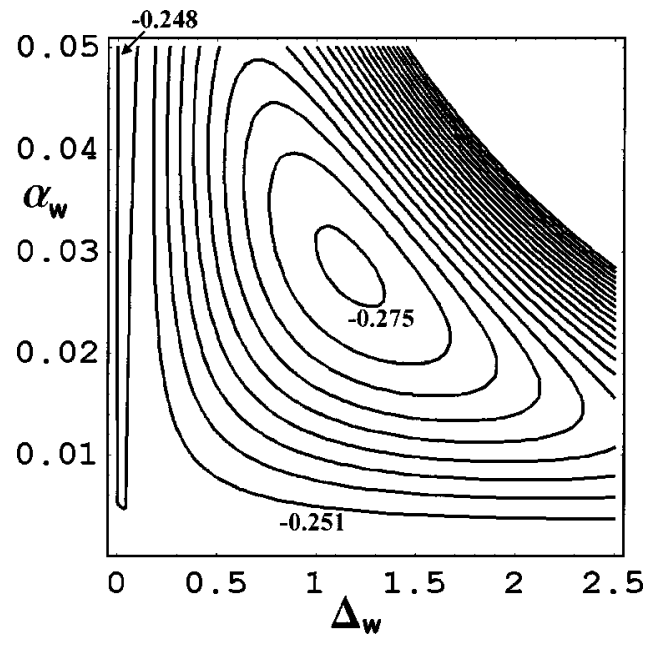

FIG. 1. Contour plot of $E_{S}\left(\alpha_{w}, \Delta_{w}\right)$ against $\alpha_{w}$ and $\Delta_{w}$. The contours are for $-0.275 \mathrm{eV}$ to $-0.215 \mathrm{eV}$, in steps of $0.003 \mathrm{eV}$. The minimum is at $\alpha_{w}=0.02840$ a.u. (a.u. stands for atomic units) and $\Delta_{w}=1.1612$ a.u. and has the value $-0.2755 \mathrm{eV}$. Note that this is higher than the actual minimum of $-0.2760 \mathrm{eV}$. This happens because we have constrained polarization to have only the form of Eq. (15).

$$
E_{S}\left(\alpha_{w}, \Delta_{w}\right)=\min _{\alpha, \beta} E\left(\alpha, \beta, \alpha_{w}, \Delta_{w}\right)
$$

Plots of this energy, as functions of $\alpha_{w}$ and $\Delta_{w}$ are given in Fig. 1. The parameters used in the plot are discussed later on in this section. If $\Delta_{w}<(1 /(8 \pi))$, then the electron has no bound state in the potential of the polarization in Eq. (15) (see Appendix B 2, for proof). For a fixed value of $\alpha_{w}$, as one increases $\Delta_{w}$, the energy at first increases, because of the increase in the energy of the polarizations. Beyond $\Delta_{w}$ $=1 /(8 \pi)$, the bound state exists and leads to a lowering of the energy. Therefore, at fixed $\alpha_{w}, E_{S}\left(\alpha_{w}, \Delta_{w}\right)$, increases at first, reaches a maximum, and then decreases. The height of the maximum depends on the value of $\alpha_{w}$ and is roughly proportional to $\alpha_{w}^{2}$. There is no such maximum along the line with $\alpha_{w}=0$. For the set of parameters given for which the plots are made in Fig. 1, the increase in energy in the region $\Delta_{w}<(1 /(8 \pi))$ can be seen in the behavior of the contour plot for the $-0.248 \mathrm{eV}$, marked in the figure. Further, note that the point $\left(\alpha_{w}, \Delta_{w}\right)=(0,0)$ is a saddle point, as is clear from the plot.

The behavior of the multidimensional surface for $\mathcal{E}$ near the delocalized state is analyzed in Appendix B 1.

Our approach may appear to treat the polarization at a classical level and the electron at the quantum level. However, after having found the extrema on the potential energy surface, one can proceed to treat the polarization modes quantum mechanically. The major effect of the quantum treatment would be to add a zero-point energy to $E(\alpha, \beta)$, and thus modify the total energy of the system. There will also be a relatively smaller effect, coming from the nonadiabatic interaction between the electrons and the polarization. However, the polarization modes are rather slow and hence this interaction is expected to have only a small effect. As far as relative energies between states are concerned, the zeropoint energies exactly cancel each other, and hence relative

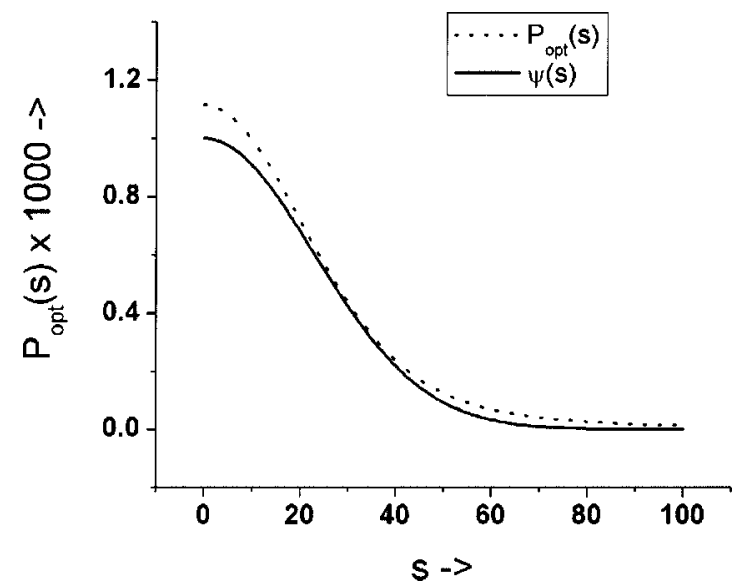

FIG. 2. Plot of the polarization, in atomic units, against the radial distance (in atomic units) from the center of the wave function. The radial dependence of the wave function also is shown. The radial part of the wave function is scaled suitably so as to fit well into the plot. Note that the polarization extends further out than the wave function.

energy differences calculated with $E(\alpha, \beta)$ would be the same as the results obtained by treating polarization modes too quantum mechanically.

We now use the above expressions to estimate the solvation properties experimentally observed for butyronitrile. ${ }^{4}$ For this, we need values of the parameters in the above equations. Using the size of butyronitrile, and assuming that the molecules are closely packed, we estimate $a$ to be $7.2 \AA$. The value of $h$ is taken to be $3.1 \AA$, so that the energy of the electron would be $-0.2481 \mathrm{eV}$, if there was no polarization in the adsorbed layer. The experiment shows the time scale of the solvation to be a few hundred femtoseconds. The experimental data of Harris et al. ${ }^{4}$ can be reproduced reasonably well if we take the dimensionless quantity $\rho \omega_{0}^{2}$ to be 1.70. Using this value and Eq. (13), we can find the potential energy surface. The contour plots in Fig. 1 show that there are two extrema in the potential and there is no barrier separating the two, which means that one of them has to be a saddle point. The minimum value of energy is $-0.2760 \mathrm{eV}$. It occurs at $\alpha=0.04211$ a.u. and $\beta=0.2034$ a.u. Converted to angstroms, $\alpha=1 /(12.56 \AA)$ and looking at Fig. 5 of Ref. 4 , this value is in reasonable agreement with experiment. The optimum polarization of Eq. (12) may be Fourier transformed to get the polarization in real space,

$$
\begin{aligned}
P_{\mathrm{opt}}(s) & =\frac{1}{2 \pi} \int d \boldsymbol{k} P_{\mathrm{opt}}(k) \exp (-i \boldsymbol{k} \cdot s) \\
& =\int_{0}^{\infty} d k k P_{\mathrm{opt}}(k) J_{0}(k s) .
\end{aligned}
$$

In the above, $s=|s|$ and $J_{0}(k s)$ denotes the Bessel function of order zero. In Fig. 2, the optimum polarization and the wave function are plotted against the radial distance $s$ from the center of the localized state.

If photoemission occurs from any of these two possible states (the extrema), then the energy of the initial state, $E_{I}$ is given by Eq. (13) and is equal to $\frac{1}{2} \alpha_{0}^{2}+\epsilon_{\mathrm{el}}\left(\beta_{0}\right)-V\left(\alpha_{0}, \beta_{0}\right)$ $+\epsilon_{\text {photon }}$, where $\left(\alpha_{0}, \beta_{0}\right)$ are the values at the extremum from which photoemission occurs and $\epsilon_{\text {photon }}$ is the energy of 
the photon. The photoemitted state would still have the nonequilibrium polarization, whose energy is easily found using Eq. (9) to be $V\left(\alpha_{0}, \beta_{0}\right)$. So the kinetic energy of emitted electron, $\epsilon_{\text {kin }}$ is given by

$$
\epsilon_{\mathrm{kin}}=\frac{1}{2} \alpha_{0}^{2}+\epsilon_{\mathrm{el}}\left(\beta_{0}\right)-2 V\left(\alpha_{0}, \beta_{0}\right)+\epsilon_{\mathrm{photon}}
$$

On calculating $\epsilon_{\text {kin }}$ for the localized and the delocalized states, with the above mentioned parameters, we find that the difference in kinetic energies of the emitted electron between the two states is $0.082 \mathrm{eV}$, which again is in fair agreement with the experiment. Another interesting point is that, if one changed the value of the dimensionless parameter $\rho \omega_{0}^{2}$, then one can change the size of the localized state continuously from small to large, which implies that the localization in this system need not be to a single lattice site, in contrast to the purely two-dimensional situation. ${ }^{4,15-17}$

An important model for polarons is the Holstein model, ${ }^{9-12}$ in which the electron has local interaction with a collection of (usually dispersionless) phonons. Within this model, the existence of a barrier for the formation of the self-trapped state in one, two and three dimensions has been studied by Kabanov and Mashtakov. ${ }^{16}$ They find that in one dimension, there is no barrier and that the self-trapped minimum is the ground state. In two dimensions, the kinetic energy of localization to a wave packet of width $R=1 / \alpha$ needs an energy $\sim \alpha^{2}$. The interaction energy with the phonons, for large sized wave packets, also behaves like $\sim \alpha^{2}$, though for smaller sized packets discreteness of the lattice becomes important and hence the behavior is different. They find the existence of three critical coupling strengths, $g_{c 1}, g_{c 2}$, and $g_{c 3}$. Depending on the value of the coupling constant $g$ one can have four different cases. They are: (1) If the coupling strength $g<g_{c 1}$, then only the delocalized state exists. (2) If $g_{c 1}<g<g_{c 2}$, then a small polaron state exists, but its energy is above that of delocalized, and is separated from it by a barrier, and hence is metastable. (3) For $g_{c 2}<g<g_{c 3}$, the trapped state is the more stable state, and the barrier still exists. (4) If $g_{c 3}<g$, the barrier disappears and the trapped state is the ground state. In the case (3) as a barrier exists between the two, the self-trapping is activated. The activation energy will be in general dependent on the initial momentum of the electron (see, for example, Ref. 7). In comparison to these, in our model, the interaction between the electron and the polarization modes is long-ranged. In spite of this, the interaction energy, for a sufficiently large wave packet is still $\sim \alpha^{2}$ (see Appendix B 1). Further, the trapped state is more stable than delocalized and as there is no barrier, the behavior of the system for the parameter values appropriate to the experiment, resembles case (4), discussed above. As the trapping is not activated even for zero momentum, one does not expect any barrier for the case with finite momentum too.

The effective mass: We now calculate the effective mass of the self-trapped state. For simplicity, we do not consider the phonon part of the wave function. Assuming a square lattice with lattice sites labeled by $\mathbf{n}=\left\{n_{x}, n_{y}\right\}, n_{x}, n_{y}$ being integers, and denoting the electronic wave function of the form of Eq. (8) located at the cite $\mathbf{n}$ as $\psi_{\mathbf{n}}$, we construct a
Bloch state of the form $\Sigma_{\mathbf{n}} \exp (i \mathbf{k} . \mathbf{n}) \psi_{\mathbf{n}} \cdot{ }^{13,14}$ We calculate the expectation value of the electronic Hamiltonian for this to be

$$
\mathcal{E}(\mathbf{k})=\frac{H_{00}+2 H_{01} \cos \left(k_{x} a\right)+2 H_{01} \cos \left(k_{y} a\right)}{1+2 S \cos \left(k_{x} a\right)+2 S \cos \left(k_{y} a\right)},
$$

with $H_{00}=\left\langle\psi_{0}\left|H_{\mathrm{el}}\right| \psi_{0}\right\rangle$ and $H_{01}=\left\langle\psi_{0}\left|H_{\mathrm{el}}\right| \psi_{1}\right\rangle$ and $S$ $=\left\langle\psi_{0} \mid \psi_{1}\right\rangle$, where $\psi_{0}$ is located on the site $\mathbf{0}$ and $\psi_{1}$ on a neighboring site. Then we have $H_{00}=\alpha^{2} / 2, \quad H_{01}$ $=-\frac{1}{8} e^{-\left(a^{2} \alpha^{2}\right) / 4} \alpha^{2}\left(-4+a^{2} \alpha^{2}\right)$ and $S=e^{-\left(a^{2} \alpha^{2}\right) / 4}$. Using these, and expanding $\mathcal{E}(\mathbf{k})$ as a series in $\mathbf{k}$, we get the effective mass to be

$$
m^{*}=\frac{4\left(2+e^{a^{2} \alpha^{2} / 4}\right)^{2} e^{-a^{2} \alpha^{2} / 4}}{a^{4} \alpha^{4}} .
$$

Using the values of $a$ and $\alpha$ for the trapped state, we get $m^{*} \approx 250$ a.u., showing that effective mass of the trapped state is quite large and hence its mobility is small. It is to be noted that the introduction of the phonon cloud into the description will only increase the effective mass.

We now discuss the dynamics briefly and this will be analyzed in detail in a later publication. The electron is initially photoexcited to a delocalized state, which however, has a finite but large size. This means that the parameter $\alpha$ is not zero, but close to zero. As the wave function is still not fully extended in space, it causes reorientation of the dipoles, but rather slowly, which is reflected by the fact that $\alpha=0$ is a saddle point, where the derivatives of $E(\alpha, \beta)$ vanish. But at the same time, the wave function begins to contract in the direction perpendicular to the surface, and the value of $\beta$ would start to increase. The portions of the wave function that have got away from the saddle point would evolve faster. As the energy is transferred to the phonons, the average energy of the electron would decrease. After a long time, the energy would reach the lowest possible value, corresponding to the trapped state, and the electron would be in this state, until it eventually escapes into the metal.

\section{CONCLUSIONS}

In this paper, we have given a continuum description of the solvation of an electron in the image state by a layer of polar adsorbates on the surface of a metal. With such a model, it is easy to make an analysis of self-trapping of the electron. The most important parameter in the self trapping is $\rho \omega_{0}^{2}$ (in atomic units, while in usual systems of units this will equal to $\rho a_{0} \omega_{0}^{2}, a_{0}$ being the radius of Bohr orbit). We find that (1) If $\rho a_{0} \omega_{0}^{2}>8 \pi$, there is no localized state and delocalized state is a minimum in the PES. Therefore, selftrapping is not possible. (2) For $\rho a_{0} \omega_{0}^{2}<4 \pi\left(2-a_{0} / a\right)$, a localized state would exist and is the stable minimum. The delocalized state would also exist, and it is not a stable minimum, but a saddle point. Hence self-trapping is a barrierless process. (3) For $4 \pi\left(2-a_{0} / a\right)<\rho a_{0} \omega_{0}^{2}<8 \pi$ the delocalized state is a stable minimum and a localized state may or may not exist. If it exists, then it too is a minimum and there would be a barrier between the two, as result of which the self-trapping is an activated process. Unlike the twodimensional case, we find that by varying the parameters, it is possible to change the size of the trapped state continu- 
ously. The model can explain the salient features of the experimental results of Harris et al. ${ }^{4}$ At the parameter value required to fit the experimental data (viz., $\rho a_{0} \omega_{0}^{2}=1.70$ ), the self-trapping is barrierless. The effective mass of the selftrapped state is quite large, being $\sim 250$ times the mass of the electron.

\section{APPENDIX A: THE DISCRETE VERSION OF THE HAMILTONIAN}

In this Appendix, we give a more detailed justification of the Hamiltonian that we use, assuming that the polarization occurs due to reorientational motion of the adsorbates, which is assumed to be rotational. Imagine that the dipolar molecules are arranged periodically on a square lattice, at lattice positions, labeled by the two dimensional vector $\boldsymbol{n}$. The lattice spacing is taken to be $a$. Let each one of them have a moment of inertia $I$ and let us imagine that the component of the dipole moment of the molecule in the $Z$-direction is zero. Rotation of the molecule can make the component in the $Z$-direction nonzero. The rotations that are caused by the electron are rather small and we shall denote the angle of rotation away from the horizonal direction by $\theta$. Further, the interaction with the surface would, in all likelihood, convert them into vibrational motions, and hence we have kinetic and potential energy contributions to the energy equal to $\frac{1}{2} \Sigma_{n} I \dot{\theta}_{n}^{2}+\frac{1}{2} \Sigma_{n} I \omega_{0}^{2} \theta_{n}^{2}$. In this, $I$ denotes the moment of inertia of the molecule for rotational motion, that will bring the molecular dipole perpendicular to the surface. In addition, the molecules will interact with the electric field at the surface, which is in the perpendicular direction. If $E_{n}$ is the electric field at the site $\boldsymbol{n}$, then the interaction energy is given by $-\Sigma_{n} E_{n} \mu \theta_{n}$, where $\mu \theta_{n}$ is the magnitude of the dipole moment of the molecule [if it rotates by an angle $\theta_{n}$, then the dipole moment in the $Z$-direction that results is $\mu \sin \left(\theta_{n}\right) \approx \mu \theta_{n}$, as $\left.\theta_{n} \ll 1\right]$. The dipoles in the $Z$-direction would interact with one another, and hence there is the contribution $\frac{1}{2} \sum_{\boldsymbol{n} \neq \boldsymbol{n}^{\prime}} \mu \theta_{\boldsymbol{n}}\left(1 / r_{\boldsymbol{n} n^{\prime}}\right)^{3} \mu \theta_{\boldsymbol{n}^{\prime}}$. Further, the dipoles would interact with their images, and this leads to a contribution $\quad \frac{1}{2} \Sigma_{\boldsymbol{n} \neq \boldsymbol{n}^{\prime}} \mu \theta_{\boldsymbol{n}}\left\{\left(1 /\left(r_{\boldsymbol{n} n^{\prime}}^{2}+d^{2}\right)\right)^{3 / 2}-\left[3 d^{2} /\left(r_{n n^{\prime}}^{2}\right.\right.\right.$ $\left.\left.\left.+d^{2}\right)^{5 / 2}\right]\right\} \mu \theta_{n^{\prime}}$, where $d$ is the distance between the plane of the dipoles and the plane of their images. In addition, there is a self-image interaction of each dipole. We shall assume that this has been included by renormalizing the value of $\omega_{0}$. Putting all these terms together, one gets

$$
\begin{aligned}
E= & \frac{1}{2} \sum_{n} I \dot{\theta}_{n}^{2}+\frac{1}{2} \sum_{n} I \omega_{0}^{2} \theta_{n}^{2}-\sum_{n} E_{n} \mu \theta_{n} \\
& +\frac{1}{2} \sum_{n \neq n^{\prime}} \mu \theta_{n}\left(\frac{1}{r_{n n^{\prime}}}\right)^{3} \mu \theta_{n^{\prime}} \\
& +\frac{1}{2} \sum_{n \neq n^{\prime}} \mu \theta_{n}\left\{\left(\frac{1}{r_{n n^{\prime}}^{2}+d^{2}}\right)^{3 / 2}\right. \\
& \left.-\frac{3 d^{2}}{\left(r_{n n^{\prime}}^{2}+d^{2}\right)^{5 / 2}}\right\} \mu \theta_{n^{\prime}} .
\end{aligned}
$$

Now we define dipole moment per unit area, $P_{n}=\mu \theta_{n} / a^{2}$, position of the $\boldsymbol{n}$ th molecule, $\boldsymbol{s}=a \boldsymbol{n}$, and $\rho=I a^{2} / \mu^{2}$. One can arrive at a simple Hamiltonian, by allowing the thickness of the dipolar layer approach zero (i.e., $d / 2 \rightarrow 0$ ), and taking the continuum limit, by putting $a^{2} \Sigma_{n} \rightarrow \int d s$, and one gets the expressions given in Eqs. (1) and (6). It is to be noted that one can also analyze the case where $d$ is finite, without much difficulty. However, we wish to keep the expression for energy as simple as possible while capturing the essential physics of the problem, and hence we use the expressions in the paper. While we have assumed rotational motion to be responsible for polarization, the same kind of derivation can be easily carried out, even in the case where it is due to vibrational motion. Thus if we denote the vibrational mode for the $\boldsymbol{n}$ th molecule as $x_{\boldsymbol{n}}$, the energy can be written as

$$
\begin{aligned}
E= & \frac{1}{2} \sum_{n} m \dot{x}_{n}^{2}+\frac{1}{2} \sum_{n} m \omega_{0}^{2} x_{n}^{2}-\sum_{n} E_{n} x_{n} \mu_{x} \\
& +\frac{1}{2} \sum_{n \neq n^{\prime}} x_{n} \mu_{x}\left(\frac{1}{r_{n n^{\prime}}}\right)^{3} \mu_{x} x_{n^{\prime}} \\
& +\frac{1}{2} \sum_{n \neq n^{\prime}} x_{n} \mu_{x}\left\{\left(\frac{1}{r_{n n^{\prime}}^{2}+d^{2}}\right)^{3 / 2}\right. \\
& \left.-\frac{3 d^{2}}{\left(r_{n n^{\prime}}^{2}+d^{2}\right)^{5 / 2}}\right\} \mu_{x} x_{n^{\prime}} .
\end{aligned}
$$

In the above $\mu_{x}=(d \mu / d x)_{x=0}$. Defining the polarization now by $P_{n}=\mu_{x} x_{n} / a^{2}$ and going over to the continuum limit leads to Eq. (1) with $\rho=m\left(a / \mu_{x}\right)^{2}$.

\section{APPENDIX B: NATURE OF THE PES}

\section{Analysis of $E(\alpha, \beta)$}

As $\mathcal{E}$ of Eq. (11) is a functional of the polarizations, the potential energy surface is infinite dimensional. However, its extrema coincide with those of $E(\alpha, \beta)$ of Eq. (13), which makes the analysis easy. It is easy to see that $0<V(\alpha, \beta)$ $\leqslant 4 a \pi \int_{0}^{\infty} d k k\left[e^{a k-2 h k} /\left(2 \pi+a e^{a k} \rho \omega_{0}^{2}\right)\right]<\infty$. Consequently, $E(\alpha, \beta) \rightarrow \infty$ as either $\alpha$ or $\beta$ approaches infinity. To find the extrema, one has to solve the two equations,

$$
\alpha=\frac{\partial V(\alpha, \beta)}{\partial \alpha}
$$

and

$$
\frac{\partial \epsilon_{\mathrm{el}}(\beta)}{\partial \beta}=\frac{\partial V(\alpha, \beta)}{\partial \beta} .
$$

On evaluation, we find

$$
\frac{\partial V(\alpha, \beta)}{\partial \alpha}=4 \pi \int_{0}^{\infty} d k \frac{k^{3}}{\alpha^{3}}\left(\frac{\beta}{k+\beta}\right)^{6} \frac{e^{-2 h k-\left(k^{2} / 2 \alpha^{2}\right)}}{\left(4 \pi e^{-a k} / a+\rho \omega_{0}^{2}\right)}
$$

and

$$
\frac{\partial V(\alpha, \beta)}{\partial \beta}=24 \pi \int_{0}^{\infty} d k k^{2} \frac{\beta^{5}}{(k+\beta)^{7}} \frac{e^{-2 h k-\left(k^{2} / 2 \alpha^{2}\right)}}{\left(4 \pi e^{-a k} / a+\rho \omega_{0}^{2}\right)} .
$$

Equations (B1) and (B2) are too complicated to find analytical solutions. However, it is possible to find the number and the nature of extrema of $E(\alpha, \beta)$ from these two equations. First we consider a plot of the left-hand and right-hand sides 


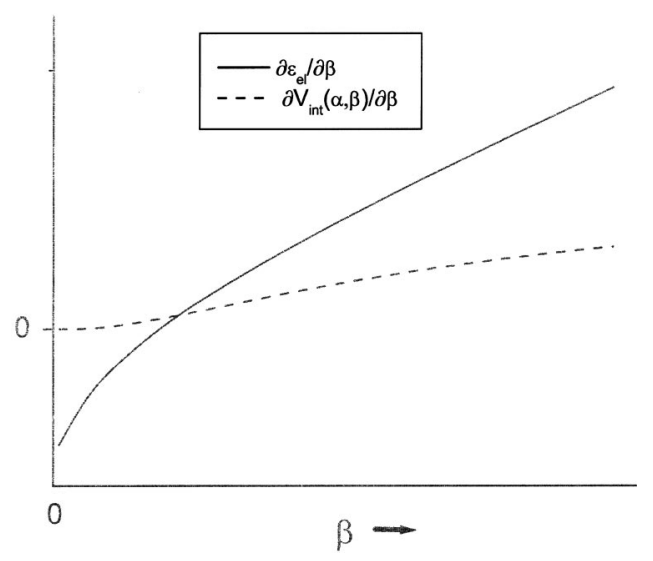

FIG. 3. Plot of $\partial V_{\text {int }}(\alpha, \beta) / \partial \beta$ against $\beta$.

of Eq. (B2). For any values of $a, h, \alpha$, and $\rho \omega_{0}^{2}$, the righthand side of the equation is an increasing function of $\beta$. Hence for any value of $\alpha$ and for any set of values of $a, h$, and $\rho \omega_{0}^{2}$ this equation has only one solution (see Fig. 3). Now we consider Eq. (B1). In Fig. 4, we make typical plots of $\partial V(\alpha, \beta) / \partial \alpha$, showing the situations that may arise. We note that for very small values of $\alpha$, only $k \sim \alpha$ contribute to the integral and so

$$
\begin{aligned}
\frac{\partial V(\alpha, \beta)}{\partial \alpha} & \simeq 4 \pi \int_{0}^{\infty} d k \frac{k^{3}}{\alpha^{3}} \frac{e^{-\left(k^{2} / 2 \alpha^{2}\right)}}{\left(4 \pi / a+\rho \omega_{0}^{2}\right)} \\
& =\frac{8 \pi}{\left(4 \pi / a+\rho \omega_{0}^{2}\right)} \alpha, \text { for } \quad \alpha \ll 1 .
\end{aligned}
$$

Thus the plot of $\partial V(\alpha, \beta) / \partial \alpha$ against $\alpha$ starts out with a slope $8 \pi /\left(4 \pi / a+\rho \omega_{0}^{2}\right)$, independent of the parameter $h$. Further, for any finite values of $\alpha$ and $\beta$,

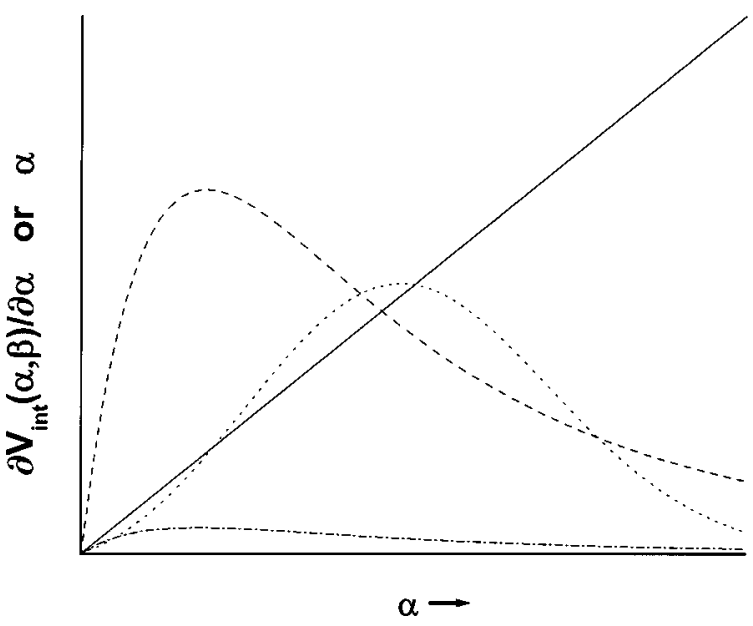

FIG. 4. (1) Straight line: $\alpha$ against $\alpha$. (2) Dashed curve: $\partial V_{\text {int }}(\alpha, \beta) / \partial \alpha$ against $\alpha$ for $8 \pi /\left(2 \pi / a+\rho \omega_{0}^{2}\right)>1$. (3) Dotted curve: $\partial V_{\text {int }}(\alpha, \beta) / \partial \alpha$ against $\alpha$ for $8 \pi>\rho \omega_{0}^{2}>8 \pi-2 \pi / a$ and the curve is assumed to intersect the $\alpha$ straight line. (4) Dotted-dashed curve: $\partial V_{\text {int }}(\alpha, \beta) / \partial \alpha$ against $\alpha$ for $\rho \omega_{0}^{2}>8 \pi$.

$$
\begin{aligned}
\frac{\partial V(\alpha, \beta)}{\partial \alpha} & =4 \pi \int_{0}^{\infty} d k \frac{k^{3}}{\alpha^{3}}\left(\frac{\beta}{k+\beta}\right)^{6} \frac{e^{-2 h k-k^{2} / 2 \alpha^{2}}}{\left(4 \pi e^{-a k} / a+\rho \omega_{0}^{2}\right)} \\
& \leqslant 4 a \pi \int_{0}^{\infty} d k \frac{k^{3}}{\alpha^{3}} \frac{e^{-2 h k-k^{2} / 2 \alpha^{2}}}{\left(4 \pi e^{-a k} / a+\rho \omega_{0}^{2}\right)} \\
& \leqslant 4 \pi \int_{0}^{\infty} d k \frac{k^{3}}{\alpha^{3}} \frac{e^{-k^{2} / 2 \alpha^{2}}}{\rho \omega_{0}^{2}}=\frac{8 \pi}{\left(\rho \omega_{0}^{2}\right)} \alpha .
\end{aligned}
$$

Thus

$$
\frac{\partial V(\alpha, \beta)}{\partial \alpha} \leqslant \frac{8 \pi}{\left(\rho \omega_{0}^{2}\right)} \alpha .
$$

Therefore the plot of $\partial V(\alpha, \beta) / \partial \alpha$ against $\alpha$ always lies below the straight line having slope $8 \pi / \rho \omega_{0}^{2}$. Further, as $\alpha$ $\rightarrow \infty, \partial V_{\text {int }}(\alpha, \beta) / \partial \alpha \rightarrow 0$. From these, we arrive at the following situations:

(1) The value of $\rho \omega_{0}^{2}>8 \pi$. This means $8 \pi / \rho \omega_{0}^{2}<1$ and in this case $\partial V(\alpha, \beta) / \partial \alpha<\alpha$ for all nonzero values of $\alpha$. Therefore, the curve and $\partial V(\alpha, \beta) / \partial \alpha$ vs $\alpha$ (dotted-dashed curve in Fig. 4) would meet the straight line $\alpha$ vs $\alpha$ only at $\alpha=0$. So Eq. (B1) has only this solution. Putting $\alpha=0$, in Eq. (B2), we find that $\partial V(\alpha, \beta) / \partial \beta=0$. This means that the electron is completely delocalized and that there is no polarization induced in the layer. The solution of Eq. (B2) then just gives the value of $\beta$ that one would expect for an electron, which is unperturbed by the polarization and moves in the image potential and bounded by the hard wall. At this point, clearly $\epsilon_{\mathrm{el}}^{\prime}(\beta)=d \epsilon_{\mathrm{el}}(\beta) / d \beta=0$. Let us denote this value of $\beta$ by $\beta_{d}$ (subscript $d$ stands for delocalized). Now we analyze the stability of this state, with $(\alpha, \beta)=\left(0, \beta_{d}\right)$, with respect to arbitrary fluctuations in the polarization. This can be very conveniently done, using the function $E(\alpha, \beta)$ as it has the interesting property that for given $(\alpha, \beta)$, it gives the variation of $\mathcal{E}$ along the path that decreases $\mathcal{E}$ the fastest. We expand $E(\alpha, \beta)$ as a series in $\alpha$ (assumed to be very small) and variations $\delta \beta$, small fluctuations in $\beta$ around $\beta_{d}$. Using the result of Eq. (B3) in Eq. (13), we find

$$
\begin{aligned}
E\left(\alpha, \beta_{d}+\delta \beta\right)= & \epsilon_{\mathrm{el}}\left(\beta_{d}\right)+\frac{1}{2} \epsilon_{\mathrm{el}}^{\prime \prime}\left(\beta_{d}\right) \delta \beta^{2} \\
& +\frac{1}{2}\left(1-\frac{8 \pi}{\left(4 \pi / a+\rho \omega_{0}^{2}\right)}\right) \alpha^{2}
\end{aligned}
$$

for infinitesimal values of $\alpha$. The condition $\rho \omega_{0}^{2}>8 \pi$ implies that $\alpha=0$ is a minimum in the $\alpha$ direction.

(2) $8 \pi-4 \pi / a>\rho \omega_{0}^{2}$. That is, $8 \pi /\left(4 \pi / a+\rho \omega_{0}^{2}\right)>1$. In this case, there are two solutions to Eq. (B1) (see the dotted curve in Fig. 4). The curve and the straight line representing the left hand side of Eq. (B1), would intersect at two points, one of them being $\alpha=0$. So $\alpha=0$ is still an extremum, but then, Eq. (B5) implies that the potential would decrease as one goes in the $\alpha$ direction. So in this direction the point is a maximum and not a minimum. As in case (1) above, it is a minimum in the $\beta$ direction and so this point is a saddle point.

(3) $8 \pi>\rho \omega_{0}^{2}>8 \pi-4 \pi / a$. The plot of $\partial V(\alpha, \beta) / \partial \alpha$ (dotted curve in Fig. 4) starts out with a slope less than unity 
and so the curve is below the straight line $\alpha$, for small values of $\alpha$. We distinguish two different cases. (a) In the first case, the curve crosses the straight line for higher values of $\alpha$, and there are three solutions to $\alpha$ for Eq. (B1). If this happens (it depends on the values of $a, h$, and $\rho \omega_{0}^{2}$ ) then the delocalized state (point $\alpha=0$ ) is a minimum and there is one more minimum, which is the localized state. Finally, the third solution has to be a saddle point and hence in this case, the localized and delocalized states are separated by a barrier. (b) In the second case, the crossing does not happen, and then there is only one solution and this is $\alpha=0$, which would then be a minimum. ${ }^{20} \mathrm{~A}$ plot in this case would resemble the dotted-dashed curve in Fig. 4 and hence is not shown separately.

\section{The surface $E_{S}\left(\Delta_{w}, \alpha_{w}\right)$}

We now look at the potential energy surface corresponding to $E_{S}\left(\alpha_{w}, \Delta_{w}\right)$ defined in the Eq. (18). We start with Eqs. (16) and (17). In the limit of infinitesimal $\alpha_{w}$, it is only very small values of $k$ that will contribute to the integral. Evaluating the energy up to second order in $\alpha_{w}$, and remembering that $\alpha_{w} \sim \alpha$, we get

$$
\begin{aligned}
E\left(\alpha, \beta, \alpha_{w}, \Delta_{w}\right)= & \frac{1}{2} \alpha^{2}+\epsilon_{\mathrm{el}}(\beta)+\frac{\pi \Delta_{w}^{2}}{4} \int_{0}^{\infty} k d k \\
& \times\left(4 \pi / a+\rho \omega_{0}^{2}\right) \exp \left(-k^{2} /\left(2 \alpha_{w}^{2}\right)\right) \\
& -2 \pi \Delta_{w} \int_{0}^{\infty} k d k \\
& \times \exp \left\{-\frac{k^{2}}{4}\left(\frac{1}{\alpha^{2}}+\frac{1}{\alpha_{w}^{2}}\right)\right\},
\end{aligned}
$$

which gives

$$
\begin{aligned}
E\left(\alpha, \beta, \alpha_{w}, \Delta_{w}\right)= & \frac{1}{2} \alpha^{2}+\epsilon_{\mathrm{el}}(\beta) \\
& +\frac{\alpha_{w}^{2} \pi \Delta_{w}^{2}}{4}\left(4 \pi / a+\rho \omega_{0}^{2}\right) \\
& -4 \pi \Delta_{w} \frac{\alpha^{2} \alpha_{w}^{2}}{\alpha^{2}+\alpha_{w}^{2}} .
\end{aligned}
$$

On finding the $\alpha$, that minimizes this energy, we get two solutions, that are physically meaningful. They are: (1) $\alpha$ $=0$ and (2) $\alpha=\alpha_{w} \sqrt{8 \pi \Delta_{w}-1}$. The second solution represents bound state of the electron in the potential well and exists only if $\Delta_{w}>1 /(8 \pi)$. We find the energy for this solution to be

$$
\begin{aligned}
E_{b}\left(\beta, \alpha_{w}, \Delta_{w}\right)= & \epsilon_{\mathrm{el}}(\beta)+\alpha_{w}^{2}\left(-1 / 2+2 \sqrt{2 \pi \Delta_{w}}\right. \\
& \left.+\pi \Delta_{w}\left\{\Delta_{w}\left(4 \pi / a+\rho \omega_{0}^{2}\right)-16\right\} / 4\right) .
\end{aligned}
$$

The above expression shows that for small values of $\alpha_{w}$, $E_{b}\left(\beta, \alpha_{w}, \Delta_{w}\right)$ is a quadratic in $\alpha_{w}$, which means that as one moves away from $\alpha_{w}=0$, the energy increases with a slope equal to zero. For certain values of $\Delta_{w}$ the coefficient of $\alpha_{w}^{2}$ is positive and that means the delocalized state is stable, while for other values of $\Delta_{w}$, the coefficient is negative and this means that the trapped state is favored. However, if $\left(4 \pi / a+\rho \omega_{0}^{2}\right)>8 \pi$, then the coefficient of $\alpha_{w}^{2}$ is positive for all values of $\Delta_{w}>1 /(8 \pi)$ and hence the delocalized state is stable, a conclusion which is in agreement with the results of Appendix B 1.

While the condition $\Delta_{w}>1 /(8 \pi)$ for the existence of a bound state stated above, has been derived in the limit $\alpha_{w}$ $\rightarrow 0$, we can easily show that this has to be obeyed for any $\alpha_{w}$ as follows. Minimizing the energy in Eq. (16) with respect to $\alpha$, we get the equation

$$
\alpha=g(\alpha)
$$

with

$$
\begin{aligned}
g(\alpha)= & \frac{\pi \Delta_{w}}{\alpha^{3}} \int_{0}^{\infty} d k k^{3} \exp \left\{-\frac{k^{2}}{4}\left(\frac{1}{\alpha^{2}}+\frac{1}{\alpha_{w}^{2}}\right)-h k\right\} \\
& \times\left(\frac{\beta}{k+\beta}\right)^{3} .
\end{aligned}
$$

Now as $h$ and $\beta$ are both positive,

$$
\begin{aligned}
g(\alpha) & \leqslant \frac{\pi \Delta_{w}}{\alpha^{3}} \int_{0}^{\infty} d k k^{3} \exp \left\{-\frac{k^{2}}{4}\left(\frac{1}{\alpha^{2}}+\frac{1}{\alpha_{w}^{2}}\right)\right\} \\
& =8 \pi \Delta_{w} \frac{\alpha \alpha_{w}^{4}}{\left(\alpha^{2}+\alpha_{w}^{2}\right)^{2}} \leqslant 8 \pi \Delta_{w} \alpha .
\end{aligned}
$$

Hence, if $8 \pi \Delta_{w}$ is less than unity, then Eq. (B7) has only $\alpha=0$ as the solution. This means that there is only the delocalized state, and no bound state.

${ }^{1}$ P. Echenique and J. Pendry, Prog. Surf. Sci. 32, 111 (1990).

${ }^{2}$ T. Fauster and W. Steinmann, in Photonic Probes of Surfaces, edited by P. Halevi (Elsevier, Amsterdam, 1995).

${ }^{3}$ R. M. Osgood, Jr. and X. Wang, Solid State Physics (Academic, San Diego, 1997), Vol. 51, p. 1.

${ }^{4}$ A. Miller, I. Bezel, K. Gaffney, S. Garrett-Roe, S. Liu, P. Szymmanski, and C. Harris, Science 297, 1163 (2002).

${ }^{5}$ K. Gaffney, A. Miller, S. H. Liu, and C. Harris, J. Phys. Chem. 105, 9031 (2001).

${ }^{6}$ N. Ge, C. Wong, and C. Harris, Acc. Chem. Res. 33, 111 (2000).

${ }^{7}$ N. Ge, C. Wong, R. Lingle, J. McNeill, K. Gaffney, and C. Harris, Science 279, 202 (1998)

${ }^{8}$ C. Harris, N. Ge, R. Lingle, J. McNeill, and C. Wong, Annu. Rev. Phys. Chem. 48, 711 (1997)

${ }^{9}$ T. Holstein, Ann. Phys. (N.Y.) 8, 325 (1959).

${ }^{10}$ T. Holstein, Ann. Phys. (N.Y.) 8, 343 (1959).

${ }^{11}$ T. Holstein, Ann. Phys. (N.Y.) 21, 494 (1963).

${ }^{12}$ T. Holstein, Ann. Phys. (N.Y.) 53, 439 (1969).

${ }^{13}$ Y. Toyozowa, Prog. Theor. Phys. 26, 29 (1961).

${ }^{14} \mathrm{G}$. Venzl and S. Fischer, Phys. Rev. B 32, 6437 (1985).

${ }^{15}$ H. Sumi and A. Sumi, J. Phys. Soc. Jpn. 63, 637 (1994).

${ }^{16}$ V. Kabanov and O. Mashtakov, Phys. Rev. B 47, 6060 (1993).

${ }^{17}$ G. Kalosakas, S. Aubry, and G. Tsironis, Phys. Rev. B 58, 3094 (1998).

${ }^{18}$ M. Tachiya, Y. Tabata, and K. Oshima, J. Phys. Chem. 77, 263 (1973).

${ }^{19}$ M. Tachiya and H. Watanabe, J. Chem. Phys. 66, 3056 (1977).

${ }^{20}$ In all the cases that we have tried, the crossing did not happen. The crossing does not seem likely in any physically meaningful range of values for the parameters $h$ and $\rho \omega_{0}^{2}$ and hence the delocalized to localized transition does not seem to be activated in most cases. 